\title{
Synthesis of $\mathrm{Sr}$ substituted $\mathrm{BaTiO}_{3}$ nanoparticles by hydrothermal treatments with maintaining the source material form and particle size
}

\author{
Hidetoshi MIYAZAKI ${ }^{1, \dagger}$, Katsumi YAMADA ${ }^{1}$, Yuta KITANO ${ }^{1}$, Yuki MAKINOSE ${ }^{1}$, Takeshi KUBOTA ${ }^{1}$, \\ Kohji OMATA ${ }^{1}$ and Shinya TSUKADA ${ }^{2}$ \\ ${ }^{1}$ Graduate School of Natural Science Technology, Shimane University, 1060 Nishikawatsu, Matsue 690-8504, Japan \\ ${ }^{2}$ Faculty of Education, Shimane University, Matsue 690-8504, Japan
}

Ion exchange of $\mathrm{Ba}$ to $\mathrm{Sr}$ was carried out for spherical $\mathrm{BaTiO}_{3}$ particles (particle size of 600 to $700 \mathrm{~nm}$ ) by hydrothermal method using strontium nitrate aqueous solution, and the hydrothermal conditions were at $200{ }^{\circ} \mathrm{C}$ for 0.5 to $1.5 \mathrm{~mol} / \mathrm{L}$ for $24 \mathrm{~h}$. After hydrothermal treatment, all samples remained the form and particle size of the source materials. The $\mathrm{Sr}$ substituted $\mathrm{BaTiO}_{3}$ sample obtained by hydrothermal treatment with strontium nitrate at $1.5 \mathrm{~mol} / \mathrm{L}$ showed the composition of $\mathrm{Sr}_{0.14} \mathrm{Ba}_{0.86} \mathrm{TiO}_{3}$ by elemental analysis by EDX, and the Curie temperature was confirmed to be $90^{\circ} \mathrm{C}$ by Raman spectra, respectively.

(C2021 The Ceramic Society of Japan. All rights reserved.

Key-words : $\mathrm{BaTiO}_{3}$, Raman spectrum, Curie temperature, lon exchange

[Received October 22, 2020; Accepted December 21, 2020]

\section{Introduction}

$\mathrm{BaTiO}_{3}$ (hereinafter, BT) has a high dielectric constant and is widely used in multi layered ceramic capacitors ${ }^{1)}$ and ferroelectric random access memories. $\left.{ }^{2}\right)$ The Curie temperature of BT is approximately $130{ }^{\circ} \mathrm{C},{ }^{3)}$ and exhibits a paraelectric property with a cubic structure at a temperature of $T_{\mathrm{c}}$ or higher, and exhibits a ferroelectric property with a tetragonal structure at a temperature of $T_{\mathrm{c}}$ or lower. ${ }^{4)}$ Because a temperature dependence of a relative permittivity of BT shows a sharp peak near $T_{\mathrm{c}}$, thereby; the addition of a shifter agent such as $\mathrm{Sr}^{5)}$ or a depressor agent such as $\mathrm{La}^{6}$ ) reduces the Tc and temperature dependence.

BT can control the Curie temperature $T_{\mathrm{c}}$ and the temperature dependence of the dielectric constant by substituting $\mathrm{Sr}$ for $\mathrm{Ba}$ in $\mathrm{BT}^{5}{ }^{5}$ ( $\left.\mathrm{Ba}, \mathrm{Sr}\right) \mathrm{TiO}_{3}$ in which $\mathrm{Sr}$ is substituted into BT is synthesized by a solid-state reaction method, a hydrothermal method, ${ }^{7)}$ a sol-gel method, ${ }^{8)}$ and so on. The solid-phase reaction method makes it easy to control the composition, but it is difficult to control the particle size and form. In addition, although it is possible to synthesize nano-sized particles with the various fabrication methods, it is difficult to remove impurities such as metal residues. In previous, we have reported the application of a hot water treatment or a hydrothermal treatment to remove $\mathrm{Na}$ from glass, ${ }^{9}$ and to exchange $\mathrm{Ca}$ ion to $\mathrm{Na}$ ion in bentonite clay. ${ }^{10)}$ On the other hand, the diffusion of $\mathrm{Sr}$ in $\mathrm{BaTiO}_{3}$ has been studied at temperatures as high as $1200^{\circ} \mathrm{C} .{ }^{11)}$ Therefore, based on our previous reports, we expected ion

Corresponding author: H. Miyazaki; E-mail: miya@riko. shimane-u.ac.jp exchange $\mathrm{Sr}$ to $\mathrm{Ba}$ in $\mathrm{BaTiO}_{3}$ by hydrothermal treatment (soft chemical process) with maintaining the source material form and particle size.

In this study, we tried to exchange $\mathrm{Ba}$ and $\mathrm{Sr}$ in $\mathrm{BT}$ while maintaining the particle size and shape by hydrothermal treatment of monodispersed BT particles in a strontium nitrate aqueous solution. The $\mathrm{Sr}$ substitution amount was evaluated from the lattice constant and elemental analysis, and the Tc of the Sr doped BT sample obtained by Raman spectrum measurement was estimated.

\section{Experimental procedure}

Strontium nitrate $\mathrm{Sr}\left(\mathrm{NO}_{3}\right)_{2}$ (FUJIFILM Wako Pure Chemical Corp., Japan) and $\mathrm{BaTiO}_{3}$ (BT-05, mean particle size $660 \mathrm{~nm}$, Sakai Chemical Industry Co.Ltd., Japan) were used as starting materials. Strontium nitrate was added to distilled water to prepare a 0.5 to $2.0 \mathrm{~mol} / \mathrm{L}$ strontium nitrate aqueous solution. This strontium nitrate aqueous solution of $80 \mathrm{~mL}$ and $\mathrm{BaTiO}_{3}$ of $1.0 \mathrm{~g}$ were mixed in a Teflon container and carried out a hydrothermal treatment at $200^{\circ} \mathrm{C}$. The treated suspension was centrifuged at 3500 $\mathrm{rpm}$ for $15 \mathrm{~min}$, and the obtained powder was dried at $60^{\circ} \mathrm{C}$ for $12 \mathrm{~h}$. This centrifugaion was repeated twice to wash the strontium nitrate adhering to the sample.

The crystal structure of the sample was measured using an X-ray diffractometer (XRD, Rigaku Miniflex, Rigaku, Japan) with $\mathrm{Cu} K \alpha$ radiation $(30 \mathrm{kV}, 15 \mathrm{~mA})$. The morphorogy of the particles was observed using a scanning electron microscope (SEM, JCM-6000, JEOL, Japan). Raman spectrum measurement was performed by a single monochromator (Lucir, Japan) with a $532 \mathrm{~nm}$ line from an $\mathrm{Nd}$ :YAG laser at RT $-550^{\circ} \mathrm{C}$ and the instruments described 
in the previous report. ${ }^{12)}$ The composition of the specimens was performed using an energy dispersive fluorescent X-ray analyzer (Shimadzu EDX-700HS).

\section{Results and discussion}

Commercial $\mathrm{BaTiO}_{3}$ (BT) particles were hydrothermal treated at $200^{\circ} \mathrm{C}$ for $24 \mathrm{~h}$ with varying strontium nitrate concentration. When the treatment temperature was less than $200^{\circ} \mathrm{C}$, almost no ion exchange was observed. A few ion-exchange was observed in the treatment times of $12 \mathrm{~h}$ or less, and the sample treated for $40 \mathrm{~h}$ or more was close to the sample treated for $24 \mathrm{~h}$.

Figures 1 and 2 illustrate the XRD patterns for the powders synthesized by hydrothermal treatment with varying the strontium nitrate concentration between 0.5 to 2.0 mol/L. Figure 1 presents the spectrum of $2 \theta$ in a wide angle $\left(10-70^{\circ}\right)$, and Fig. 2 represents the spectrum around (110) $(011)$ peaks $\left(31.2-32.0^{\circ}\right)$. All the powders assigned as tetragonal $\mathrm{BaTiO}_{3}$ with JCPDS 83-1875. From Fig. 1, it was confirmed that the structure of BT was not changed by the hydrothermal treatment and that strontium nitrate residue was not observed in the hydrothermal-treated powders. From Fig. 2, it was observed that the (110) and (101) peaks were shifted to higher angle with increase of the strontium nitrate concentration. From the XRD pattern, the peak (Fig. 2) is slightly obscured in the BST sample. We will discuss this result later, but $\mathrm{Sr}$ ions assumed not to be sufficiently diffused into the BT particles. Ota et al. reported that the XRD peak shifted to a wide angle with increase of Sr-doped amount in $(\mathrm{Ba}, \mathrm{Sr}) \mathrm{TiO}_{3} .{ }^{13)}$ Our results agreed well with the reported results. These results suggested that $\mathrm{Ba}$ in $\mathrm{BaTiO}_{3}$ was substituted to $\mathrm{Sr}$ and that the amount of Sr substitution changed depending on the strontium nitrate concentration. For the powder treated with strontium

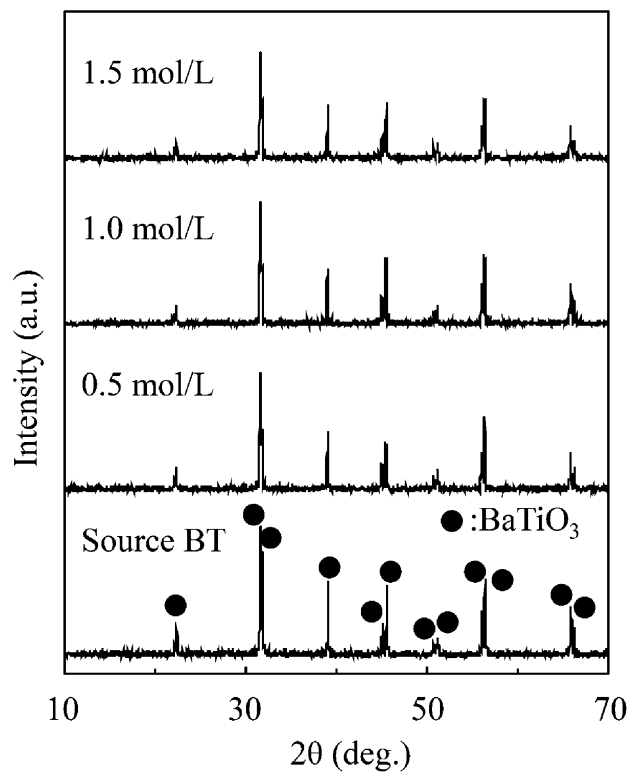

Fig. 1. The XRD patterns for the source $\mathrm{BaTiO}_{3}$ powder and the hydrothermal treated $\mathrm{BaTiO}_{3}$ powders using strontium nitrate aqueous solution with the concentration of $0.5-1.5 \mathrm{~mol} / \mathrm{L}$. The $2 \theta$ angle was between 10 to $70^{\circ}$. nitrate concentration of $2.0 \mathrm{~mol} / \mathrm{L}$ or more, the XRD peak was almost the same as that of the sample treated with 1.5 mol/L concentration. Because the XRD peak shift was the largest in the powder hydrothermal treated with the strontium nitrate of $1.5 \mathrm{~mol} / \mathrm{L}$, we performed SEM observation, Raman evaluation, and composition analysis by EDS for this sample. Hereafter, we call this sample BST in this paper.

Figure 3 depicts the SEM images of the source BT powder and the hydrothermal treated BST powder. The source BT powder was spherical form and had a mean particle diameter of $660 \mathrm{~nm}$, and BST after the hydrothermal treatment was also spherical form and the mean particle diameter was $630 \mathrm{~nm}$. As can be seen from the SEM image, the particle form was almost unchanged before and after the hydrothermal treatment, and the particle size was also maintained. These results revealed that hydrothermal treatment can substitute $\mathrm{Ba}$ to $\mathrm{Sr}$ while maintaining the form and size of the $\mathrm{BaTiO}_{3}$ particles.

Figure 4 demonstrates the results of Raman spectra for BT and BST powders at room temperature. The spectra of those were in good agreement with the Raman spectra of $\mathrm{BaTiO}_{3}$ samples reported in previous investigations. ${ }^{14), 15)}$ For the Raman spectrum signal of $\mathrm{BaTiO}_{3}$, Dobal et al. have reported that the intensity of $\mathrm{E}(\mathrm{TO} 1)$ mode decreases drastically above the Curie temperature $T_{\mathrm{c}} \cdot{ }^{15)} \mathrm{We}$ evaluated the temperature dependence of E(TO1) mode in Raman spectra of BT and BST in order to estimate the $T_{\mathrm{c}}$ of those powders. The inset in Fig. 4 also demonstrates the temperature dependence of E(TO1) mode of Raman spectra of $\mathrm{BT}$ and BST. The intensity of the $\mathrm{E}(\mathrm{TO} 1)$ mode decreased significantly at about $130^{\circ} \mathrm{C}$ for BT and at $90^{\circ} \mathrm{C}$ for BST. From these results, the $T_{\mathrm{c}}$ of BT and the obtained BST was estimated to be 130 and $90^{\circ} \mathrm{C}$, respectively. However, the intensity of $\mathrm{E}$ (TO1) was not a critical change comparison with that of the non-doped BT. The $\mathrm{Sr}$ concentration in BT particle assumed to be inhomogeneous from the XRD

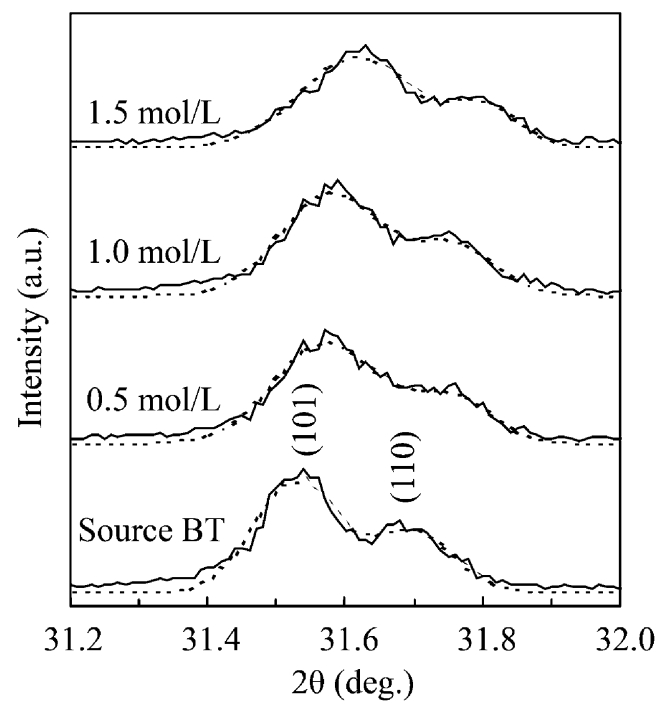

Fig. 2. The XRD patterns for the samples, ibid of Fig. 1. The $2 \theta$ angle was between 31.2 to $32^{\circ}$. 

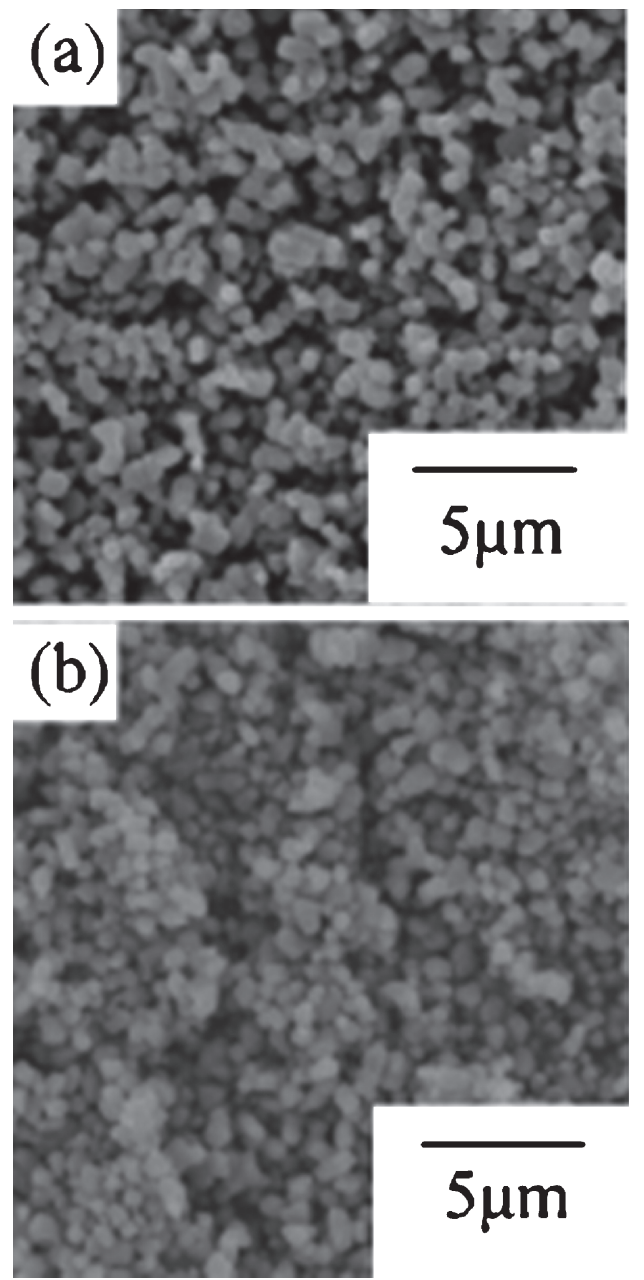

Fig. 3. The SEM images of the source BT and the resulting BST powders.

measurement results. Similarly, the moderate intensity change of the E (TO1) peak in the temperature dependence of the Raman spectra assumed to be attributed to inhomogeneity of Sr distribution in the BT particles. It was confirmed that the $T_{\mathrm{c}}$ of the hydrothermal treated BST powder decreased about $40^{\circ} \mathrm{C}$. It was suggested that ion exchange $\mathrm{Ba}$ in BT to $\mathrm{Sr}$ was caused by hydrothermal treatment, and the composition of the hydrothermal treated BST powder was estimated to be $\mathrm{Sr}_{0.11} \mathrm{Ba}_{0.89} \mathrm{TiO}_{3}$ from the result of $T_{\mathrm{c}}$ decrease. ${ }^{15)}$ The elemental analysis of the BST sample by EDS revealed $\mathrm{Sr}_{0.14} \mathrm{Ba}_{0.86} \mathrm{TiO}_{3}$, and the composition calculated from Raman spectroscopy was slightly smaller than the elemental analysis value. We also evaluated the lattice constant from the XRD measurement for the BST samples, and the lattice constants of the BST were $a=3.993 \AA$, $c=4.030 \AA$. In previous, the correlation between the substitution amount $x$ in $\mathrm{Sr}_{x} \mathrm{Ba}_{1-x} \mathrm{TiO}_{3}$ and the lattice constant of $\mathrm{Sr}_{x} \mathrm{Ba}_{1-x} \mathrm{TiO}_{3}$ has been reported. ${ }^{16)}$ We calculated the composition from the previous report and the lattice constants in the present study and the composition was $\mathrm{Sr}_{0.03} \mathrm{Ba}_{0.97} \mathrm{TiO}_{3}$. The composition calculated from this lattice constant was smaller than those obtained from EDS and Raman results. Sr diffusion into the BT particles was
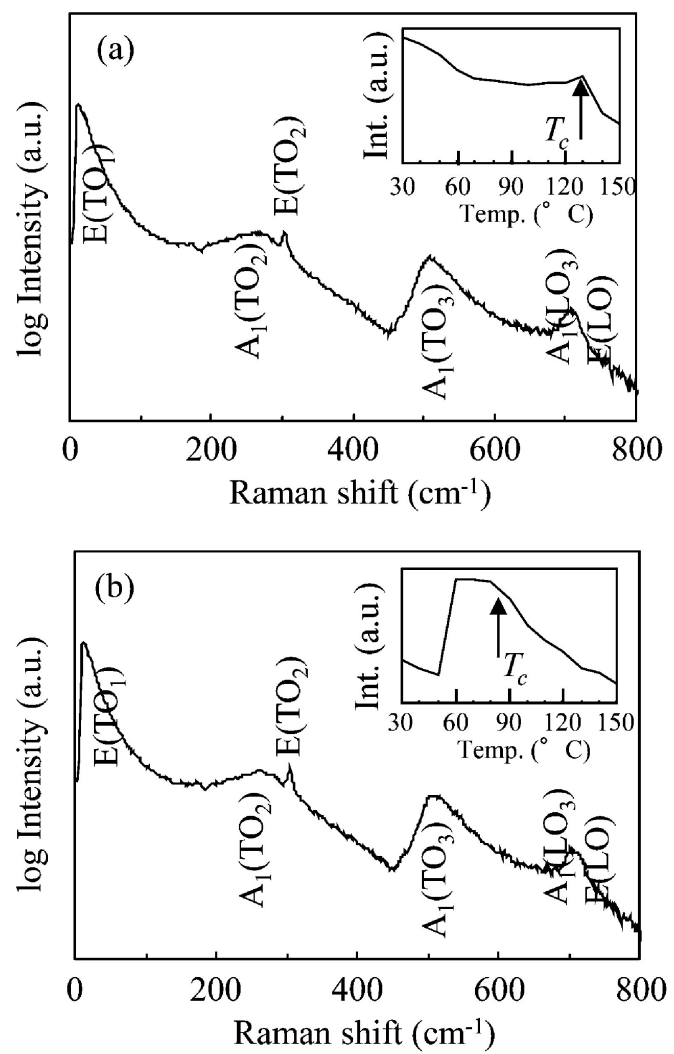

Fig. 4. The Raman spectra for the source BT and the resulting BST powders.

assumed to be insufficient. Furthermore, because BST was synthesized by $\mathrm{Sr}$ diffusion into BT under hydrothermal conditions, the composition of the particle surface and the particle center assumed to be different slightly. Therefore, it shall be necessary to study hydrothermal conditions such as solvent and temperature in the future works.

\section{Conclusion}

We successfully synthesized Sr-doped $\mathrm{BaTiO}_{3}$ by $\mathrm{BaTiO}_{3}$ nanoparticles to hydrothermal treatment using an strontium nitrate aqueous solution, and the particle size (around $600 \mathrm{~nm}$ ) and the form (spherical) of the source $\mathrm{BaTiO}_{3}$ were maintained after hydrothermal treatment. It was confirmed that $\mathrm{Ba}$ in $\mathrm{BT}$ was substituted $\mathrm{Sr}$ from the composition analysis by EDS and temperature dependence of Raman spectra. In conclusion, it was proved that Srsubstituted $\mathrm{BaTiO}_{3}$ particles could be easily obtained by hydrothermal treatment of source $\mathrm{BaTiO}_{3}$ spherical nanoparticles (particle size of sub hundreds nanometer) with maintaining the form and particle size of the source material. Furthermore, ion exchange assumed to be possible by extending this method for materials those undergo phase changes due to slight composition changes and materials with various shapes such as needle-shaped particles and thin films.

\section{References}

1) C. Pithan, D. Hennings and R. Waser, Int. J. Appl. Ceram. Tec., 2, 1-14 (2005). 
2) J. F. Scott, Ferroelectrics Rev., 1, 1-129 (1998).

3) L. Zhou, P. M. Vilarinho and J. L. Baptista, J. Eur. Ceram. Soc., 19, 2015-2020 (1999).

4) H. Hayashi, T. Nakamura and T. Ebina, J. Phys. Chem. Solids, 74, 957-962 (2013).

5) M. Arshad, D. Huiling, J. M. Sufyan, A. Maqsood, I. Ashraf, S. Hussain, W. Ma and H. Ran, Ceram. Int., 46, 2238-2246 (2020).

6) M. T. Buscaglia, V. Buscaglia, M. Viviani, P. Nanni and M. Hanuskov, J. Eur. Ceram. Soc., 20, 1997-2007 (2000).

7) T. Kato, T. Hirai, M. Mizuno, K. Kurachi and I. Taga, Ferroelectrics, 154, 331-336 (1994).

8) M. C. Gust, L. A. Momoda, N. D. Evans and M. L. Mecartney, J. Am. Ceram. Soc., 84, 1087-1092 (2001).

9) S. Yoshida, H. Miyazaki, S. Sugahara, Y. Seike, H. Suzuki and T. Ota, Mater. Cycles Waste Manage. Res., 26, 84-88 (2015).
10) H. Miyazaki, Y. Kitano, Y. Makinose, M. Handa and T. Nakashima, Clay Sci., 23, 47-53 (2019).

11) S. Koerfer, R. A. D. Souza, H.-I. Yoo and M. Martin, Solid State Sci., 10, 725-734 (2008).

12) S. Tsukada, Y. Fujii, Y. Yoneda, H. Moriwake, A. Konishi and Y. Akishige, Phys. Rev. B, 97, 024116 (2018).

13) T. Ota, Y. Abe, H. Miyazaki, T. Hirashita, K. Daimon, Y. Hikichi and H. Suzuki, J. Ceram. Soc. Jpn., 109, 174-176 (2001).

14) J. L. Parsons and L. Rimai, Solid State Commun., 5, 423-427 (1967).

15) P. S. Dobal, A. Dixit, R. S. Katiyar, D. Garcia, R. Guo and A. S. Bhalla, J. Raman Spectrosc., 32, 147-149 (2001).

16) L. Zhou, P. M. Vilarinho and J. L. Baptista, J. Eur. Ceram. Soc., 19, 2015-2020 (1999). 